\title{
Ältere erwerbstätige Frauen und gesundheitliche Ungleichheit
}

\author{
S. Tophoven
}

\section{Abstract}

Der Anteil älterer Beschäftigter ist in den letzten 20 Jahren stark gestiegen. Dies zeigt sich besonders deutlich für Frauen: 2018 sind über die Hälfte der Frauen der Altersgruppe der 60- bis unter 65-Jährigen erwerbstätig, 1998 waren es nur $11 \%$. Vor diesem Hintergrund müssen die Erwerbssituationen älterer erwerbstätiger Frauen in den Fokus rücken. Der Beitrag betrachtet die funktionale körperliche Gesundheit bei älteren erwerbstätigen Frauen in Abhängigkeit von verschiedenen Merkmalen ihrer beruflichen Tätigkeit. Dabei zeigt sich unter anderem ein klarer sozialer Gradient in Hinblick auf das Anforderungsniveau der ausgeübten Tätigkeit.

\section{1. Ältere erwerbstätige Frauen und gesundheitliche Ungleichheit}

Vor dem Hintergrund des demografischen Wandels und mit Blick auf die sozialen Sicherungssysteme bestand schon lange die Forderung nach einer längeren Erwerbstätigkeit aller und den entsprechenden Systemanpassungen (Böhle 2000). Veränderte gesetzliche Rahmenbedingungen wie die Erhöhung des gesetzlichen Renteneintrittsalters sind inzwischen in Kraft getreten. Auch aus betrieblicher Sicht zum Erhalt von Fachkräften wie auch aus individueller Perspektive hinsichtlich der Alterssicherung ist eine längere Erwerbstätigkeit aktuell angezeigt. Arbeit unter guten alter(n)sgerechten Bedingungen muss dabei für alle möglich sein (Kistler 2008, Mühlenbrock 2016).

Der demografische Wandel hat in Deutschland zu einer Alterung der Bevölkerung geführt und führt perspektivisch zu einer Schrumpfung (Kaufmann 2005). Die daraus resultierende prognostizierte Veränderung der Zusammensetzung der Erwerbsbevölkerung (Pahl 2000) ist inzwischen eingetreten. Es gibt mehr ältere Erwerbstätige. In Deutschland hat in den letzten beiden Jahrzehnten vor allem die Erwerbstätigkeit von Frauen deutlich zugenommen. Ein besonders hoher Anstieg ist für die weibliche Erwerbsbeteiligung im höheren Erwerbsalter festzustellen. Dies unter- 
streicht die Notwendigkeit einer geschlechtsspezifischen Perspektive auf das Altern, die Arbeitssituationen und die Gesundheit älterer Beschäftigter (Backes 2010, Crawford et al. 2016, Payne/Doyal 2010).

Der deutsche Arbeitsmarkt ist durch ein hohes Maß an Geschlechtersegregation gekennzeichnet. Frauen und Männer konzentrieren sich auf unterschiedliche Berufe und Frauen arbeiten seltener als Männer in Führungspositionen und häufiger in niedrigen beruflichen Positionen (Achatz 2018). Frauen arbeiten zudem häufig in Teilzeittätigkeiten (Vogel 2009). Damit ist in Teilen auch die beharrliche geschlechtsspezifische Lohnlücke zuungunsten von Frauen zu erklären (Boll/Leppin 2015). Die geringere Erwerbsbeteiligung und geringere Einkünfte im Lebensverlauf von Frauen bedingen auch eine geschlechtsspezifische Lücke in der Alterssicherung zuungunsten von Frauen (Tophoven/Tisch 2016). Im Besonderen die Erwerbsverläufe von Müttern sind häufig von Erwerbsunterbrechungen und langen Teilzeiterwerbstätigkeitsphasen geprägt, um die Vereinbarkeit von Beruf und Familie zu ermöglichen (Ziefle 2009).

Vor diesem Hintergrund betrachtet der vorliegende Beitrag die Erwerbssituationen von älteren weiblichen Beschäftigten, die der Babyboomer-Generation angehören. Nachgegangen wird der Frage, in welchen Bereichen möglicherweise gesundheitliche Ungleichheiten in Abhängigkeit von Merkmalen der beruflichen Tätigkeit bestehen. Die Erwerbssituation und Arbeitsbelastungen sind zentral zur Erklärung gesundheitlicher Ungleichheit bei Erwerbstätigen (Jungbauer-Gans/Gross 2009). Dargestellt wird die physische funktionale Gesundheit differenziert nach Berufssegmenten und dem Anforderungsniveau beruflicher Tätigkeiten sowie in Abhängigkeit von verschiedenen möglichen belastenden Faktoren der Erwerbssituation. Hieraus sollen Hinweise für den spezifischen präventiven Handlungsbedarf für die Situationen älterer erwerbstätiger Frauen gewonnen werden.

\subsection{Gestiegene Erwerbsbeteiligung von Frauen im höheren Erwerbsalter}

An dieser Stelle wird aber zunächst noch einmal die deutlich gestiegene Erwerbsbeteiligung von Frauen im höheren Erwerbsalter aufgezeigt. Dies wird sowohl zahlenmäßig wie auch anteilsmäßig deutlich. Die geburtenstarken Jahrgänge der 1960er-Jahre, die Babyboomer, sind im höheren Erwerbsalter angekommen. Abbildung 1 zeigt, dass 2018, im Vergleich zu 1998, deutlich mehr Erwerbstätige 50 bis unter 65 Jahre alt sind. 1998 waren es noch 7,8 Millionen, 2018 waren es dann 14,5 Millionen Erwerbstätige. Die Zahl hat sich also nahezu verdoppelt. Besonders deutlich wird der 
Anstieg in der Altersgruppe der 60 bis unter 65-Jährigen. 1998 gab es in dieser Altersgruppe etwa 700.000 erwerbstätige Männer und 300.000 erwerbstätige Männer, 2018 sind es 1,7 Millionen erwerbstätige Männer und 1,5 Millionen erwerbstätige Frauen. Insgesamt haben sich die Zahlen der erwerbstätigen Frauen und Männer in allen Altersgruppen deutlich angenähert (vgl. Abbildung 1).

\section{Abbildung 1: Anzabl erwerbstätiger Frauen und Männern nach Altersgruppen in Millionen - 1998 und 2018}

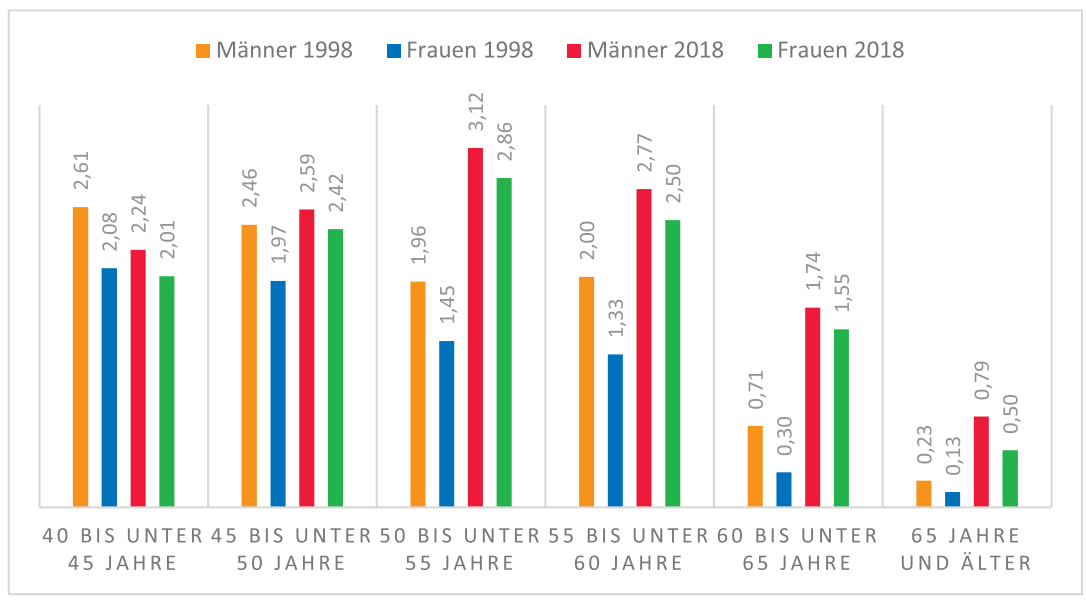

Quelle: GENESIS-Online des Statistischen Bundesamtes. Daten des Mikrozensus 1998 und 2018; eigene Darstellung und Berechnungen.

Abbildung 2 stellt die Anteile der Erwerbstätigen in den verschiedenen Altersgruppen dar.

In der Altersgruppe der 50- bis unter 55-Jährigen waren $199861 \%$ der Frauen und $82 \%$ der Männer erwerbstätig, 2018 sind es in dieser Altersgruppe $83 \%$ der Frauen und $90 \%$ der Männer. In der Altersgruppe der 55bis unter 60-Jährigen sowie in der Altersgruppe der 60- bis 65-Jährigen zeigt sich dies noch deutlicher. Hier fällt außerdem der inzwischen deutlich höhere Anteil erwerbstätiger Frauen auf. So waren in der Altersgruppe der 60- bis unter 65-Jährigen 1998 nur 11\% der Frauen erwerbstätig, 2018 sind es $56 \%$ und damit mehr als die Hälfte der Frauen dieser Altersgruppe (vgl. Abbildung 2).

Der gestiegene Anteil älterer Erwerbstätiger im Zeitverlauf ist auf die Alterung der Erwerbsbevölkerung insgesamt zurückzuführen, aber auch 
Abbildung 2: Anteile erwerbstätiger Frauen und Männer nach Altersgruppen in Prozent - 1998 und 2018

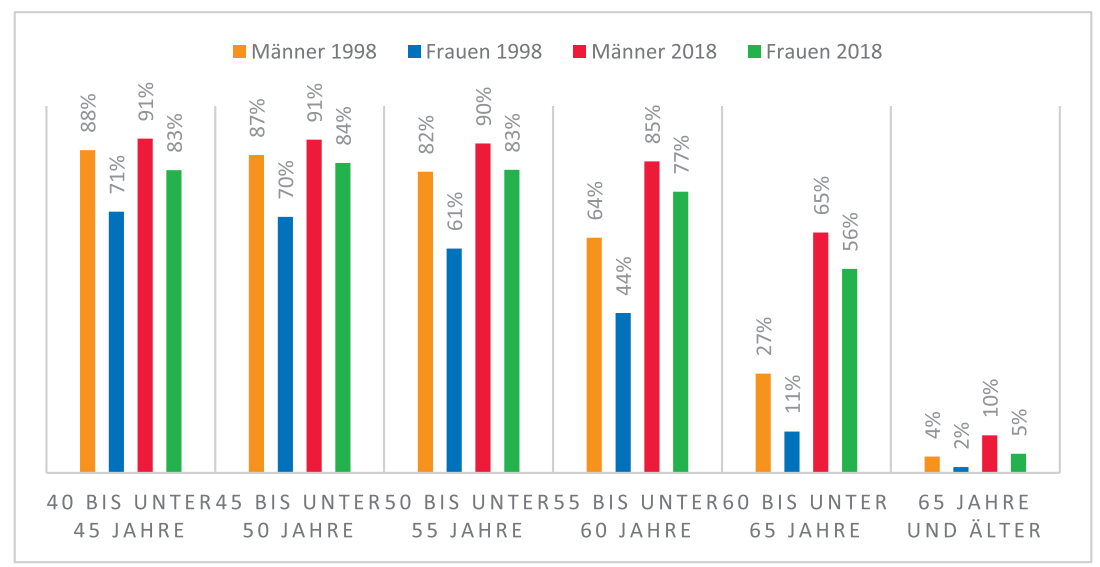

Quelle: GENESIS-Online des Statistischen Bundesamtes. Daten des Mikrozensus 1998 und 2018; eigene Darstellung und Berechnungen.

auf eine insgesamt gestiegene Erwerbsbeteiligung Älterer. Dazu tragen die veränderten gesetzlichen Rahmenbedingungen wie die schrittweise Erhöhung des gesetzlichen Renteneintrittsalters und auch das angeglichene Renteneintrittsalter von Frauen und Männern bei (Rauch et al. 2017).

Festzuhalten ist aber auch, dass sich der Erwerbstätigkeitsumfang zwischen Frauen und Männern weiterhin deutlich unterscheidet. Der starke Anstieg erwerbstätiger Frauen wird auch auf die Zunahme von Teilzeitbeschäftigungen zurückgeführt. Frauen arbeiten deutlich häufiger als Männer in Teilzeit oder in einem geringfügigen Beschäftigungsverhältnis (Statistik der Bundesagentur für Arbeit 2019).

\subsection{Gesundheitliche Situation von erwerbstätigen Frauen der Babyboomer- Generation}

Zur Beschreibung der gesundheitlichen Situation von erwerbstätigen Frauen der Babyboomer-Generation wird auf empirische Ergebnisse auf Basis der lidA-Studie (Hasselhorn 2014, Tophoven et al. 2016) zurückgegriffen. ${ }^{1}$ Herangezogen werden die Scores der funktionalen physischen Gesundheit. $^{2}$ Von den 2011 befragten erwerbstätigen Frauen im Alter von 46 und 52 Jahren waren $42 \%$ der Frauen in Vollzeit erwerbstätig und $46 \%$ in 
Teilzeit erwerbstätig. $12 \%$ übten eine geringfügige Beschäftigung aus. In Bezug auf die gesundheitliche Situation zeigen sich zwischen den unterschiedlichen Erwerbsformen nur marginale bis keine Unterschiede. Für die befragten erwerbstätigen Frauen der Geburtsjahrgänge 1959 und 1965 kann zum Befragungszeitpunkt im Mittel ein relativ guter Gesundheitsstatus festgestellt werden (Tophoven 2018). In Abhängigkeit von verschiedenen Merkmalen der beruflichen Situation lassen sich dabei aber auch einige Auffälligkeiten feststellen, die im Folgenden dargestellt werden.

\section{Funktionale Gesundheit nach Berufssegmenten}

Abbildung 3: Score funktionale physische Gesundheit in Abhängigkeit vom Berufssegment

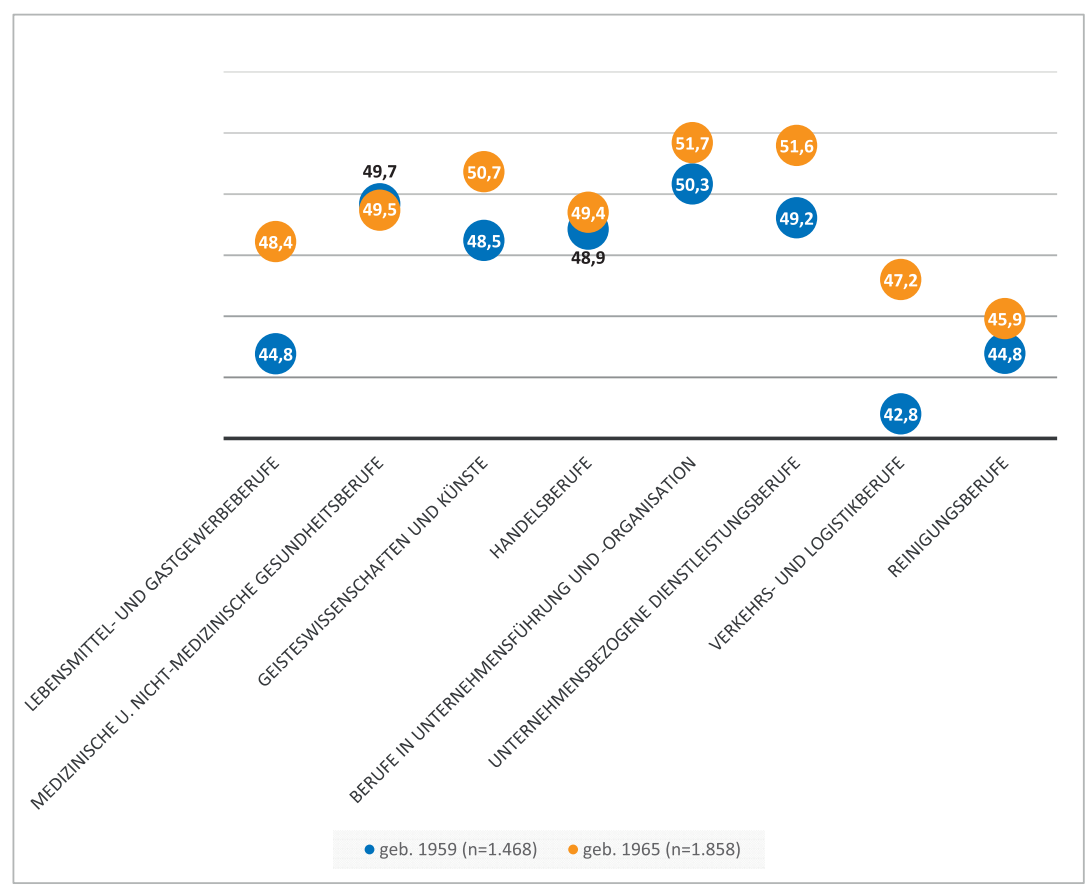

Quelle: lidA Welle 1 (2011); eigene Berechnungen

Berufssegmente, in denen die befragten Frauen häufig tätig sind, sind medizinische und nicht-medizinische Gesundheitsberufe (19\%), Berufe in 
Unternehmensführung und Unternehmensorganisation (16\%), Tätigkeiten im Bereich Geisteswissenschaft oder Kunst (15\%) sowie unternehmensbezogene Dienstleistungsberufe (14\%) und Handelsberufe (12\%). Seltener sind sie in Reinigungsberufen (5\%), Lebensmittel- und Gastgewerbeberufen (4\%) sowie Verkehrs- und Logistikberufen (4\%) tätig. Weitere $9 \%$ verteilen sich auf die Berufssegmente: Land-, Forst- und Gartenbauberufen, Fertigungsberufe, fertigungstechnische Berufe, Bau- und Ausbauberufe, IT- und naturwissenschaftliche Dienstleistungsberufe oder Sicherheitsberufe (Tophoven 2018). Die funktionale physische Gesundheit wird nun für die acht am häufigsten vorkommenden Berufssegmente dargestellt (Abbildung 3).

Im Mittel liegt die funktionale körperliche Gesundheit über alle Berufssegmente hinweg für die jüngere Kohorte bei 50,1\% (geb. 1965) und bei 48,5 \% für die ältere Kohorte (geb. 1959). Die jüngere Kohorte weist demnach im Mittel erwartbar einen etwas besseren Wert auf als die ältere Kohorte. Differenziert nach den verschiedenen Berufssegmenten, lassen sich hier teilweise größere Unterschiede feststellen. Drei Berufssegmente fallen für beide Kohorten mit durchschnittlich besonders niedrigeren Scores der funktionalen körperlichen Gesundheit auf. Dies sind Berufe im Lebensmittel- oder Gastgewerbe (zum Gastgewerbe s. Beitrag von Guhlemann \& Best in diesem Band), Verkehrs- und Logistikberufe sowie Reinigungsberufe. Eine durchschnittlich etwas bessere Gesundheit weisen in beiden Kohorten Beschäftigte in Berufen der Unternehmensführung und -organisation auf (vgl. Abbildung 3). Es zeigt sich außerdem, dass die Unterschiede zwischen den Kohorten je nach Berufssegment etwas variieren.

\section{Funktionale Gesundheit nach Anforderungsniveau der beruflichen Tätigkeit}

Die verschiedenen Berufssegmente ermöglichen noch keine Aussage über die berufliche Position innerhalb des Berufes. Dazu wird im nächsten Schritt das Anforderungsniveau der beruflichen Tätigkeit als vertikale Dimension herangezogen (Paulus/Matthes 2013). Diese Systematik unterscheidet vier Kategorien. Bei den betrachteten weiblichen Erwerbstätigen arbeitet der überwiegende Anteil in fachlich ausgerichteten Tätigkeiten (60\%). $15 \%$ üben komplexe Spezialistinnentätigkeiten aus, $13 \%$ übernehmen hoch komplexe Tätigkeiten (höchste Kategorie) und $12 \%$ verrichten Hilfs- oder Anlerntätigkeiten (niedrigste Kategorie) (Tophoven 2018). 
Abbildung 4: Score funktionale physische Gesundheit in Abhängigkeit vom Anforderungsniveau
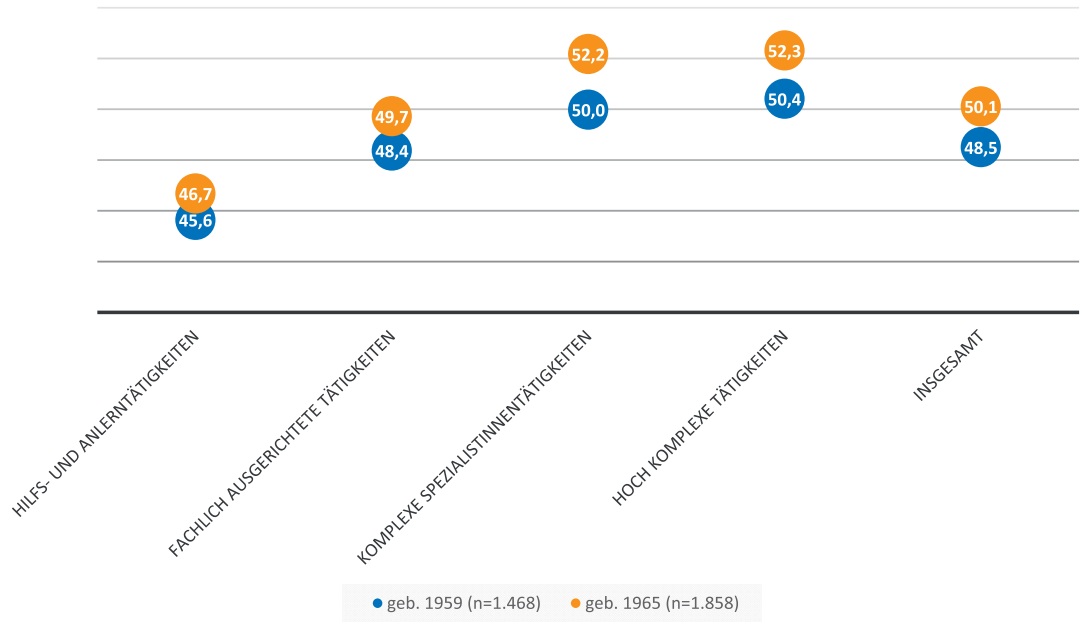

Quelle: lidA Welle 1 (2011); eigene Berechnungen

Abbildung 4 stellt die Scores der funktionalen körperlichen Gesundheit in Abhängigkeit vom Anforderungsniveau der ausgeübten Tätigkeit dar. Bereits auf den ersten Blick zeigt sich hier ein sozialer Gradient. Je höher das Anforderungsniveau der ausgeübten Tätigkeit, desto höher der Score der funktionalen physischen Gesundheit. Der Unterschied zwischen Frauen in Hilfs- und Anlerntätigkeiten und hoch komplexen Tätigkeiten beträgt 4,8 Punkte für die 52-jährigen Frauen und 5,6 Punkte für die 46-jährigen Frauen. Unterschiede zwischen den beiden Altersjahrgängen sind hier geringer und ähnlich wie in der Gesamtpopulation.

\section{Funktionale Gesundheit nach prekären Merkmalen der beruflichen Tätigkeit}

In einer weiterführenden Betrachtung wurden verschiedene Merkmale der Erwerbssituation auf ihren Zusammenhang zur funktionalen körperlichen Gesundheit hin betrachtet (Tophoven/Tisch 2016, Tophoven 2018). Abbildung 5 zeigt die Regressionsergebnisse auf den Score der funktionalen physischen Gesundheit. Als erklärende Merkmale werden verschiedene als prekär eingestufte Merkmale der Erwerbssituation herangezogen (Tophoven/Tisch 2016). 
Abbildung 5: Multivariate Betrachtung der funktionalen physischen Gesundbeit

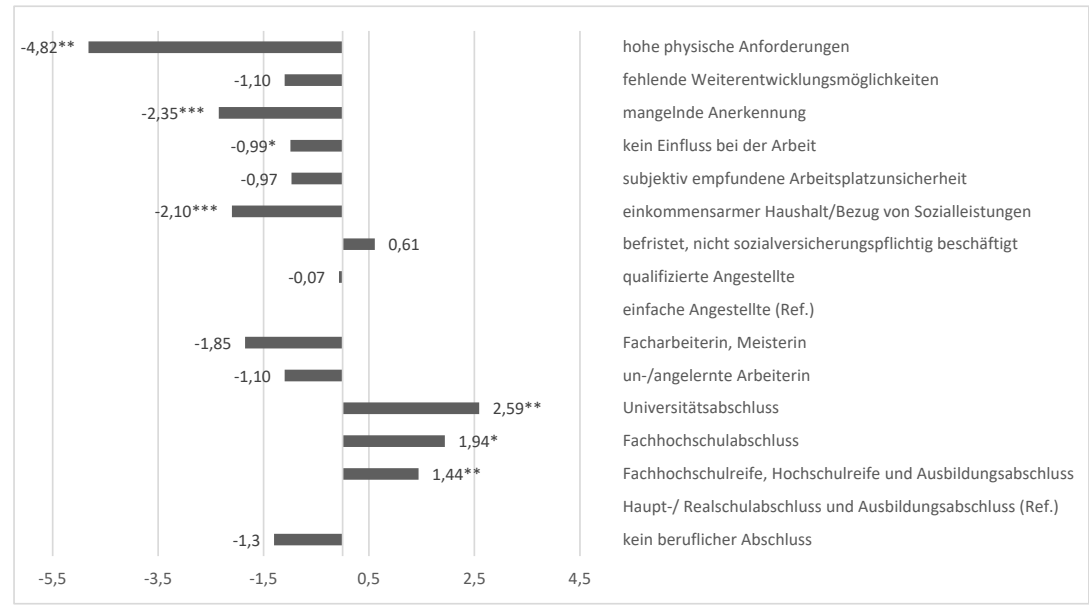

Quelle: lidA Welle 1 (2011), OLS-Regression, abhängige Variable: Score funktionale physische Gesundheit, außerdem kontrolliert für Geburtsjahr, Voll- oder Teilzeitbeschäftigung, Dauer jetzige Beschäftigung und Haushalts- bzw. Partnerschaftssituation, $n=2.760$, adj. $R^{2}=9,4 \%$; eigene Berechnungen

Hinsichtlich dieser Merkmale zeigte sich, dass eine materiell-prekäre Lebenssituation (Einkommensarmut und/oder Bezug von Sozialleistungen) und Kennzeichen der Tätigkeit wie mangelnde Anerkennung, fehlender Einfluss und hohe physische Arbeitsanforderungen in einem signifikant negativen Zusammenhang zur funktionalen körperlichen Gesundheit stehen. Daneben zeigte sich außerdem, dass ein hoher beruflicher Abschluss in einem positiven Zusammenhang zur funktionalen physischen Gesundheit steht.

\subsection{Fazit}

In den letzten 20 Jahren ist der Anteil älterer Beschäftigter deutlich gestiegen. Dies wird besonders deutlich für Frauen. So waren beispielsweise in der Altersgruppe der 60- bis unter 65-Jährigen 1998 nur $11 \%$ der Frauen erwerbstätig, 2018 sind es $56 \%$. Ihre Erwerbssituationen müssen verstärkt in den Fokus der Forschung zum gesunden Älterwerden in der Arbeit rücken. Der vorliegende Beitrag untersuchte bestehende gesundheitliche Un- 
gleichheiten bei älteren erwerbstätigen Frauen. Die Ergebnisse zeigen, dass ihr Gesundheitszustand insgesamt gut ist. In einer differenzierten Betrachtung fällt allerdings auf, dass es auch erwerbstätige Frauen gibt, die eine geringere funktionale körperliche Gesundheit berichten. Dies zeigt sich für Berufe im Bereich Lebensmittel, Gastgewerbe, Verkehr und Logistik sowie für Reinigungsberufe. Besonders deutlich wird dies bei der Betrachtung in Abhängigkeit vom Anforderungsniveau der ausgeübten Tätigkeit. Je höher das Anforderungsniveau, desto besser die funktionale körperliche Gesundheit. Eine multivariate Betrachtung verdeutlicht, dass als Erklärungsfaktoren verschiedene Belastungen in der Erwerbssituation herangezogen werden können wie ein geringes Haushaltseinkommen und tätigkeitbezogene Merkmale wie mangelnde Anerkennung und hohe physische Arbeitsanforderungen. Hier zeigt sich außerdem, dass ein hoher Ausbildungsabschluss - auch unter Kontrolle weiterer Faktoren der Erwerbssituation - mit einer höheren funktionalen Gesundheit einhergeht.

Behrens (2000) verweist bereits auf Unterschiede in der Art der Tätigkeiten und in Erwerbsverläufen die maßgeblich sind für begrenzte Tätigkeitsdauern. Er stellt heraus, dass der Art der Tätigkeit und dem Erwerbsverlauf entscheidende Rollen als Erklärungsfaktoren für die ungleichen Chancen auf eine lange Erwerbstätigkeit zukommen und weniger der individuellen Leistungsfähigkeit. Gleichzeitig macht er auch auf frühzeitige präventive Ansatzpunkte bei der Ausgestaltung von Erwerbsarbeit aufmerksam. So sollten auch vulnerable Gruppen wie Frauen in körperlich belastenden Tätigkeiten oder Tätigkeiten mit einem geringen Anforderungsniveau frühzeitig in den Fokus von Prävention und Karriereplanung rücken (vgl. hierzu auch Hiesinger/Tophoven 2019). Zimmer et al. (2010) verweisen hier u.a. auf das hohe Potenzial eines besseren Angebots und einer besseren Ausgestaltung von formalen wie non-formalen Weiterbildungen für Arbeitnehmer"innen.

Eine kontinuierliche Erwerbstätigkeit ist aktuell außerdem die zuverlässigste Quelle zur Sicherung des zukünftigen Lebensunterhalts in der Nacherwerbsphase (Zimmer et al. 2010). Vor allem Frauen sind für den Ruhestand deutlich schlechter abgesichert als Männer (Klammer 2017). Auch vor diesem Hintergrund ist eine holistische Perspektive auf die Ausgestaltung des Erwerbsverlaufs und der Erwerbssituationen von Frauen und ihren gesundheitlichen Risiken nötig. Dies ist eine zentrale Aufgabe, der sich Politik, Sozialversicherungen wie auch Betriebe und Beschäftigtenvertretungen stellen müssen. Die präventive Gestaltung von Erwerbsverläufen ist eine gemeinsame Aufgabe. Mit dem Präventionsgesetz wurde inzwischen eine Grundlage für die gemeinsame Umsetzung und stärkere $\mathrm{Zu}$ sammenarbeit geschaffen (Lampert et al. 2019). Ausreichende und passen- 
de präventive Angebote zum Erhalt und zur Förderung der Gesundheit und Kenntnisse über diese müssen allen zur Verfügung stehen.

Ausgeblendet wird im vorliegenden Beitrag die Sorgearbeit, die im privaten Bereich geleistet wird. Diese wird zu hohen Anteilen von Frauen übernommen. Auch aus dieser können sich gesundheitliche Belastungsfaktoren ergeben. Aktuell ist das Ziel einer Gleichstellung der Geschlechter noch nicht erreicht. Eine zentrale Rolle kommt dabei der Gleichstellung im Berufsleben zu, die ein Schlüssel ist, um die häufig schlechteren materiellen Positionen von Frauen auszugleichen. Dazu gehören aber auch eine Gleichstellung und gute Bedingungen bei der Sorgearbeit im privaten Bereich (Sachverständigenkommission zum Zweiten Gleichstellungsbericht der Bundesregierung 2017).

\section{Literatur}

Achatz, Juliane (2018): Berufliche Geschlechtersegregation. In: Abraham, Martin; Hinz, Thomas (Hrsg.): Arbeitsmarktsoziologie. Probleme, Theorien, empirische Befunde (3., überarb. Aufl.). Wiesbaden: Springer VS, 389-435.

Andersen, Hanfried H.; Mühlbacher, Axel; Nübling, Matthias; Schupp, Jürgen; Wagner, Gert G. (2007): Computation of Standard Values for Physical and Mental Health Scale Scores Using the SOEP Version of SF-12v2. Schmollers Jahrbuch, 127, 1, 171-182.

Backes, Gertrud M. (2010): Alter(n): Ein kaum entdecktes Arbeitsfeld der Frauenund Geschlechterforschung. In: Becker, Ruth; Kortendiek, Beate (Hrsg.): Handbuch Frauen- und Geschlechterforschung. Theorie, Methoden, Empirie. Wiesbaden: VS Verlag für Sozialwissenschaften, 454-460.

Behrens, Johann (2000): Was Demographie mit Kinder kriegen zu tun hat und was uns vorzeitig alt aussehen lässt - Illusionen im Trendmodell der Erwerbszeit. In: Von Rothkirch, Christoph (Hrsg.): Altern und Arbeit: Herausforderung für Wirtschaft und Gesellschaft. Berlin: Edition Sigma, 228-258.

Böhle, Fritz (2000): Alter und Arbeit - Erwerbsarbeit zwischen Markt und Staat als neue Herausforderung für die Arbeitsmarkt- und Sozialpolitik. In: Von Rothkirch, Christoph (Hrsg.): Altern und Arbeit: Herausforderung für Wirtschaft und Gesellschaft. Berlin: Edition Sigma, 310-338.

Boll, Christina; Leppin, Julian S. (2015): Die geschlechtsspezifische Lohnlücke in Deutschland: Umfang, Ursachen und Interpretation. Wirtschaftsdienst, 95, 4, 249-254.

Crawford, Joanne O.; Davis, Alice; Cowie, Hilary; Dixon, Ken (2016): Women and the ageing workforce. Implications for Occupational Safety and Health A research review. European Agency for Safety and Health at Work. 
Hasselhorn, Hans Martin; Peter, Richard; Rauch, Angela; Schröder, Helmut; Swart, Enno; Bender, Stefan; du Prel, Jean-Baptist; Ebener, Melanie; March, Stefanie; Trappmann, Mark; Steinwede, Jacob; Müller, Bernd Hans (2014): Cohort profile: The lidA Cohort Study-a German Cohort Study on Work, Age, Health and Work Participation. International Journal of Epidemiology, 43, 6, 1736-1749.

Hiesinger, Karolin; Tophoven, Silke (2019): Job requirement level, work demands, and health: a prospective study among older workers. International Archives of Occupational and Environmental Health, 92, November, 1139-1149.

Jungbauer-Gans, Monika; Gross, Christiane (2009): Erklärungsansätze sozial differenzierter Gesundheitschancen. In: Richter, Matthias; Hurrelmann, Klaus (Hrsg.): Gesundheitliche Ungleichheit. Grundlagen, Probleme, Perspektiven (2., aktual. Aufl.). Wiesbaden: VS Verlag für Sozialwissenschaften, 77-98.

Kaufmann, Franz-Xaver (2005): Schrumpfende Gesellschaft. Vom Bevölkerungsrückgang und seinen Folgen. Frankfurt a. M.: Suhrkamp.

Kistler, Ernst (2008): Alternsgerechte Erwerbsarbeit. Ein Überblick über den Stand von Wissenschaft und Praxis, Böckler Forschungsmonitoring 7. Düsseldorf.

Klammer, Ute (2017): Alterssicherung von Frauen revisited - aktuelle Entwicklungen und zukünftige Perspektiven. Sozialer Fortschritt, 66, 5, 359-375.

Lampert, Thomas; Hoebel, Jens; Kuntz, Benjamin; Waldhauer, Julia (2019): Soziale Ungleichheit und Gesundheit. In: Haring, Robin (Hrsg.): Gesundheitswissenschaften. Springer Reference Pflege - Therapie - Gesundheit. Berlin, Heidelberg: Springer, $155-164$.

Mühlenbrock, Inga (2016): Alterns- und altersgerechte Arbeitsgestaltung. Grundlagen und Handlungsfelder für die Praxis (1. Aufl.). Dortmund: Bundesanstalt für Arbeitsschutz und Arbeitsmedizin (BAuA).

Nübling, Matthias; Andersen, Hanfried H.; Mühlbacher, Axel (2006): Entwicklung eines Verfahrens zur Berechnung der körperlichen und psychischen Summenskalen auf Basis der SOEP-Version des SF 12 (Algorithmus), Data Documentation 16, Berlin.

Pahl, Veronika (2000): Altern und Arbeit - Chancengleichheit für alle Altersgruppen. In: Von Rothkirch, Christoph (Hrsg.): Altern und Arbeit: Herausforderung für Wirtschaft und Gesellschaft. Berlin: Edition Sigma, 13-18.

Payne, S.; Doyal, L. (2010): Older women, work and health. Occupational Medicine, 60, 3, 172-177.

Paulus, Wiebke; Matthes, Britta (2013): The German classification of occupations 2010 - structure, coding and conversion table, FDZ-Methodenreport 08/2013, Nürnberg.

Rauch, Angela; Tisch, Anita; Tophoven, Silke (2017): Erwerbsbeteiligung Älterer. In: Müller, Joachim; Walwei, Ulrich (Hrsg.): Arbeitsmarkt kompakt. Analysen, Daten, Fakten. Bielefeld: Bertelsmann, 30-31.

Sachverständigenkommission zum Zweiten Gleichstellungsbericht der Bundesregierung (Hrsg.) (2017): Erwerbs- und Sorgearbeit gemeinsam neu gestalten. Gutachten für den Zweiten Gleichstellungsbericht der Bundesregierung. Berlin. 
Statistik der Bundesagentur für Arbeit (2019): Berichte: Blickpunkt Arbeitsmarkt. Juli 2019. Die Ar-beitsmarktsituation von Frauen und Männern. Nürnberg.

Tisch, Anita; Tophoven, Silke (2012): Employment biographies of the German baby boomer generation, Schmollers Jahrbuch 132, 2, 205-232.

Tophoven, Silke (2018): Erwerbsverlauf und Gesundheit älterer weiblicher Beschäftigter. Bielefeld: wbv.

Tophoven, Silke; Tisch, Anita (2016): Dimensionen prekärer Beschäftigung und Gesundheit im mittleren Lebensalter. WSI Mitteilungen, 69, 2, 105-112.

Tophoven, Silke; Tisch, Anita (2016): Employment trajectories of German baby boomers and their effect on statutory pension entitlements. Advances in Life Course Research, 30, 2016, 90-110.

Tophoven, Silke; Wurdack, Anja; Rauch, Angela; Munkert, Casandra; Bauer, Ulrike (2016): lidA - leben in der Arbeit Kohortenstudie zu Gesundheit und Älterwerden in der Arbeit. Dokumentation für die Wellen 1 und 2. FDZ-Datenreport 01/2016 (de), Nürnberg.

Vogel, Claudia (2009): Teilzeitbeschäftigung - Ausmaß und Bestimmungsgründe der Erwerbsübergänge von Frauen. Zeitschrift für ArbeitsmarktForschung, 42, 2, 170-181.

Ziefle, Andrea (2009): Familienpolitik als Determinante weiblicher Lebensverläufe? Die Auswirkungen des Erziehungsurlaubs auf Familien- und Erwerbsbiographien in Deutschland. Wiesbaden: VS Verlag für Sozialwissenschaften.

Zimmer, Barbara; Leve, Verena; Naegele, Gerhard (2010): „Rente mit 67" - Befunde zur Erwerbssituation älterer Arbeitnehmerinnen. Comparative Population Studies - Zeitschrift für Bevölkerungswissenschaft, 35, 4, 709-738.

\section{Referenzen}

1 Der vorliegende Beitrag stellt einige zentrale empirische Ergebnisse der 2018 veröffentlichten Dissertation der Verfasserin zusammen (Tophoven 2018).

2 Diese Skala basiert auf zwölf Fragen und weist einen theoretischen Wertebereich von 0 bis 100 auf. Ein Wert von 100 entspricht einer weit überdurchschnittlich sehr guten Gesundheit. Die Daten werden üblicherweise normiert. Grundlage ist die SOEP-Population von 2004. Ein Wert von 50 entspricht damit dem Durchschnitt der SOEP-Population von 2004 (Nübling et al. 2006; Andersen et al. 2007). 